\section{Recherche clinique : Pourquoi est-elle importante en 2018}

En 2017, lors de la conférence de la Société Canadienne de Médecine Interne, j’ai eu le privilège de recevoir le Prix d'excellence David-Sackett 2018 destiné à un chercheur chevronné. Je voudrais partager avec vous quelques principaux points d'une conférence sur la recherche clinique et les raisons pour lesquelles elle garde (encore) en 2018 l'importance de cette époque. Elle s'adresse aux aspirants investigateurs cliniques et aux médecins en début de carrière qui envisagent une carrière complète ou partielle en recherche clinique, mais peuvent ne pas savoir si cela " en vaut la peine ». Pour élucider votre lecture, je m'efforcerai de vous convaincre que la recherche clinique a de l'importance et que vous décidez simplement de tremper vos orteils dans les eaux peu profondes ou de les plonger dans les profondeurs, elle enrichira votre vie et fera de vous un meilleur médecin.

D’abord, considérons les raisons de ne pas faire de la recherche une partie de vos activités professionnelles. J'ai entendu des aspirants chercheurs et même des collègues chevronnés offrir les moyens de dissuasion suivants : " Il y a trop de barrières administratives " ou " Je ne suis pas bon en écriture, en particulier en matière de subventions ». Mon préféré c'est :

"Toutes les questions importantes ont déjà été répondues ». Même si je suis d'accord qu'en 2018 la recherche clinique comprend une composante administrative plus dense (par exemple, formulaires de consentement de quinze pages contre une page) et qu'il y a plus d'obstacles à franchir (par exemple, contrats et révision juridique) qu'auparavant, d'autres possibilités de recherche ont émergé. Malgré l'accroissement du contenu administration associée à la recherche clinique, ces changements sont positifs, car ils fournissent aux patients et aux chercheurs plus de contrôles et d'équilibres pour leur permettre d'effectuer les recherches les plus rigoureuses possibles. En ce qui concerne l'affirmation selon laquelle le secteur de la recherche se rétrécit, nous pouvons le réfuter en disant que répondre à une question de recherche créera de nouvelles questions. En outre, en 2018, de nouveaux domaines de recherche, qui nétaient peut-être pas aussi visibles dans le passé, ont vu le jour. Ces recherches comprennent celles relatives aux initiatives d'amélioration de la qualité (par exemple, Efficacité de Choisir Judicieusement), celles sur les données volumineuses (par exemple, Utilisation de bases de données administratives connexes pour évaluer l'intervention et les dommages liés aux drogues) et la recherche liée à l'application des connaissances (par exemple, la question de savoir si et comment nous fournissons les soins de santé et de déterminer si cela est efficace est d'une importance capitale en 2018. En outre, ces nouveaux domaines (combinés avec les domaines de recherche clinique plus traditionnels) gagneront en popularité auprès des bailleurs de fonds lorsque l'augmentation des tensions est liée à une population vieillissante de consommateurs de soins de santé et les ressources limitées pour faire face à ces besoins croissants.

Pour les jeunes aspirants cliniciens en herbe qui font de la recherche dans le cadre de leur plan de carrière, je peux vous suggérer quelques choses à faire et à ne pas faire.
1. Vous devez penser à vos propres idées de projets : Vous êtes plus susceptible d'être engagé si vous avez la propriété du projet. N'acceptez pas rapidement que vos idées manquent de mérite : Soyez respectueux mais méfiez-vous des récalcitrants.

2. Faites confiance aux opinions de votre superviseur : Son expérience vous guidera dans la bonne voie. Ne faites pas toujours confiance aux opinions de votre superviseur : son expérience peut être biaisée en fonction de ses propres priorités.

3. Pensez grand : Soyez ambitieux dans vos objectifs; les idées ne coûtent rien et la curiosité est d'une importance primordiale. Ne pensez pas trop grand : Tempérez l'ambition avec le caractère pratique et réservez les projets les plus ambitieux pour plus tard.

4. Essayez dêtre dans un bon environnement de recherche: L'environnement permettra l'exposition à différentes opportunités. Ne vous découragez pas si vous êtes dans un petit centre : les idées et un ou deux défenseurs engagés sont importants.

5. Maintenez votre intégrité : Une fois perdue, l'intégrité est difficile à retrouver et ne vaut pas le risque d'être perdue. $\mathrm{Ne}$ brûlez pas les ponts: le monde de la recherche est petit et il n'y il ne sert à rien de contrarier les collègues ou les

institutions.

Voyons maintenant pourquoi la recherche devrait être intégrée dans votre carrière d'interniste. Pour les internistes académiques, il fournit un véhicule pour cultiver l'expertise et être à la pointe des nouveaux développements dans un domaine choisi qui, à son tour, fournira un substrat pour l'avancement d'une carrière académique. Pour les internistes communautaires, un objectif de recherche peut fournir une base pour le leadership et l'avancement administratif. En dehors de l'aspect " aller de l'avant ", une carrière qui inclut la recherche offre d'autres avantages tels que la récompense et la gratification continue de la stimulation intellectuelle et l'interaction avec des collègues à un très haut niveau. Cependant, l'avantage le plus important est son effet sur les soins de vos patients : en menant la recherche, nous posons constamment des questions sur ce que nous faisons, comment nous le faisons et pourquoi nous le faisons. Cette autoévaluation critique de la façon dont nous pratiquons la médecine - elle-même un sous-produit d'un environnement de recherche - nous permet de mieux communiquer avec nos patients et de mieux les prendre en charge.

DOI: 10.22374/cjgim.v13i2.295

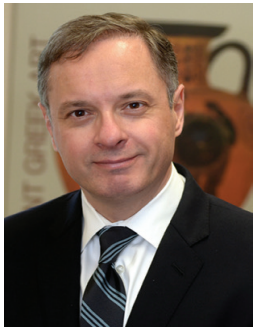

Jim Douketis, MD

Éditeur en chef 